\title{
Toward a Scalable Visualization System for Network Traffic Monitoring*
}

\author{
Erwan LE MALÉCOT ${ }^{\dagger a)}$, Masayoshi KOHARA ${ }^{\dagger \dagger}$, Nonmembers, Yoshiaki HORI ${ }^{\dagger \mathrm{b})}$, \\ and Kouichi SAKURAI ${ }^{\dagger c)}$, Members
}

\begin{abstract}
SUMMARY With the multiplication of attacks against computer networks, system administrators are required to monitor carefully the traffic exchanged by the networks they manage. However, that monitoring task is increasingly laborious because of the augmentation of the amount of data to analyze. And that trend is going to intensify with the explosion of the number of devices connected to computer networks along with the global rise of the available network bandwidth. So system administrators now heavily rely on automated tools to assist them and simplify the analysis of the data. Yet, these tools provide limited support and, most of the time, require highly skilled operators. Recently, some research teams have started to study the application of visualization techniques to the analysis of network traffic data. We believe that this original approach can also allow system administrators to deal with the large amount of data they have to process. In this paper, we introduce a tool for network traffic monitoring using visualization techniques that we developed in order to assist the system administrators of our corporate network. We explain how we designed the tool and some of the choices we made regarding the visualization techniques to use. The resulting tool proposes two linked representations of the network traffic and activity, one in 2D and the other in 3D. As 2D and $3 \mathrm{D}$ visualization techniques have different assets, we resulted in combining them in our tool to take advantage of their complementarity. We finally tested our tool in order to evaluate the accuracy of our approach.

key words: visualization, interactivity, network, monitoring, security
\end{abstract}

\section{Introduction}

Sending e-mails and browsing the Web are becoming daily life activities of an increasing number of people and the number of devices connected to the Internet is exploding. Internet is constituted of interconnected computer networks which are far from being secure and reliable. Attacks against such networks are performed around the clock (e.g. Denial of Service (DoS), spoofing, software vulnerabilities exploitation, etc.) [3]. However common Internet users are usually not aware of that underlying architecture and its issues. To minimize the risks for these users, system admin-

Manuscript received August 8, 2007.

Manuscript revised December 13, 2007.

${ }^{\dagger}$ The authors are with the Graduate School of Information Science and Electrical Engineering, Kyushu University, Fukuoka-shi, 819-0395 Japan.

${ }^{\dagger \dagger}$ The author contributed to this work when he was a graduate student at the Graduate School of Information Science and Electrical Engineering, Kyushu University.

*This is an extended paper based on the authors' previously published works at IEEE IAW'06 [1] and ACM VizSEC'06 [2] workshops.

a)E-mail: lemalecot@itslab.csce.kyushu-u.ac.jp

b)E-mail: hori@csce.kyushu-u.ac.jp

c) E-mail: sakurai@ @ csce.kyushu-u.ac.jp

DOI: 10.1093/ietisy/e91-d.5.1300 istrators need to manage and protect efficiently their networks. That task is mainly performed monitoring the traffic between connected devices by using various tools.

With the global increase of network bandwidth, the amount of data (resulting from traffic logging) that system administrators have to analyze tend to become quite huge. It is no longer possible to process these logs the classical way, by reading them directly, with tools such as Tcpdump [4] or Wireshark [5] (previously Ethereal). So system administrators now heavily rely on computer capabilities to do that processing and extract meaningful information from the traffic logs. Network Intrusion Detection Systems (NIDS) are systems based on that methodology. NIDSs check all logged network packets for suspicious patterns and generate alerts if they detect ones. However, at the moment, such systems are characterized by high rates of false positive alerts. Also, conventional NIDSs are based on databases of known attack signatures and consequently, they cannot detect unknown attacks. This is even more critical due to the increasing release of zero-day flaws by ever malicious people or the research community. Moreover, complex attack patterns can be missed by systems based solely on machines.

We believe that these limitations can be overcome by combining more efficiently human-based and machinebased processing of the data. Recently, various research teams have started to consider the use of visualization techniques in the field of network monitoring and computer security as those techniques were successfully used in various over fields (e.g. physics, biology, chemistry, etc.) to simplify the analysis of large sets of data and enhance the comprehension of complex phenomena [6]. The main idea is to take advantage of human visual processing and pattern recognition to complete machine processing. Following that trend, we decided to design a visualization system to assist system administrators in the monitoring task of our corporate network. In this paper we present the resulting visualization system along with the main steps that led us to the current system. We started by developing a tool called PGVis (Packet Grid Visualization) based on a 2D representation of the network address space on which we displayed network traffic and activity [1]. However after a period of testing, we realized that the tool had several limitations that we tried to solve by combining our $2 \mathrm{D}$ representation of the network space with a 3D one, thus upgrading the tool [2].

This paper is organized as follows. In Sect. 2, we first introduce the goals and the analysis that led us to develop 
our initial visualization design for network traffic monitoring. In Sect. 3, we present the solution we imagined to represent efficiently the network space in our different tools. In Sect. 4, we describe the first visualization that we produced (2D visualization). In Sect. 5, we present the second visualization (3D visualization) that we developed to overcome some limitations of the first one. In Sect. 6, we describe how to link the visualizations for better usability. In Sect. 7, we introduce the prototypes we built according to our visualization designs. In Sect. 8, we describe and analyze some recent work in the field of visualization techniques for network traffic monitoring and network security. Finally, in Sect. 9, we conclude on our research and discuss some leads about future work.

\section{Initial Approach}

Our primary objective was to design an efficient visualization system that would help the system administrators managing our corporate network and enable them to easily detect network anomalies. We first used our background in network security and network traffic monitoring in order to produce a preliminary set of what functionalities such a system needed to propose. We also discussed with one of the system administrator in charge of our corporate network to get his insight into the subject. That interview was really helpful as we were able to confront our ideas with the real needs of a system administrator. We extracted the following major requirements:

1. The visualization system should display the internal activity of the local network that the system administrator is in charge of.

2. The visualization system should display the interactions between that local network and the external hosts communicating with it.

3. The visualization system should display the characteristics of those interactions.

We knew what to display, we had to determine how to display it. So we analyzed thoroughly previously proposed visualization systems for network traffic monitoring. We decided to focus on systems based on a visual metaphor of the network topology because we thought that system administrators would be more incline to use that kind of system. Then the first basic requirement of such a system is a way to represent the network space and the associated hosts. Among the visualization systems we analyzed, a few provided the possibility to clearly visualize both the internal activity of the local network and the communications of that network with remote hosts. They usually focused on only one of these two functionalities. Also, none of these tools provided a way to efficiently represent all the remote hosts as their number can become really huge depending on the nature of the local network.

Another aspect is that the interaction of the user with the representation of the network space was most of the time non-existent. He was usually provided with a static view of the topology which sometimes automatically evolved according to network events. However he couldn't modify it to meet his needs, for instance focusing on a specific zone. We believe that user interaction is fundamental in visualization systems. The user should be able to actively explore the data displayed and not passively watch some pieces of information, as enunciated by Shneiderman in his visual information seeking mantra: "Overview first, zoom and filter, then details-on-demand" [7].

In addition to these comments, we wanted the position of a given host to be fixed over time on our representation of the network space. Without that constraint, it seemed quite difficult for the user of the visualization system to recognize patterns on the display as the underlying representation could change.

We tried to design a network space representation that would match all the previously proposed criteria.

\section{Interactive Grid Representation of the Network Space}

The original network space representation we imagined is hierarchical and based on interactive $16 \times 16$ and $256 \times 256$ grids (Fig. 1). Using that system, the user can access and visualize the activity of any network part, ranging from the global network to a single host. Moreover, that representation complies with our previously enunciated constraint, the position of each host and network zone being fixed once and for all.

Our hierarchical representation of the network space is composed of five layers. The grids corresponding to the first four layers are $16 \times 16$ and stand for network zones or hosts. Hosts are mapped on the grids according to their IP addresses. In the IPv4 system, IP addresses are coded by 32 bits which can be grouped in four bytes, as it is done for the dotted quad notation. In the initial state (layer one), hosts are mapped on the grids based on the first byte of their

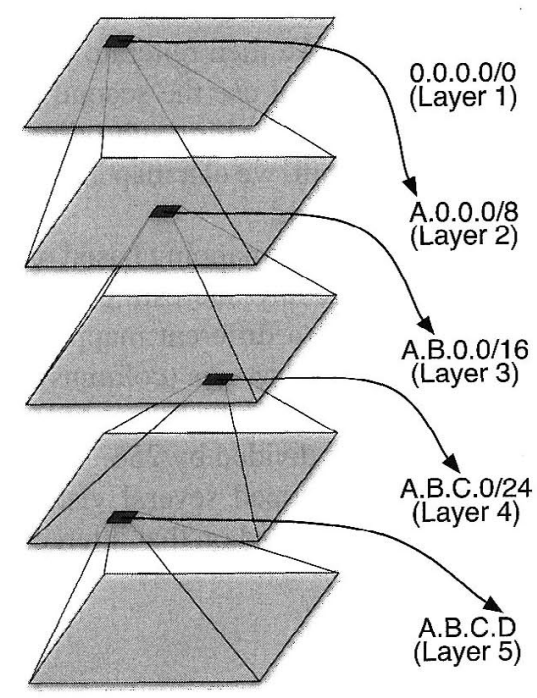

Fig. 1 Interactive grid system. 


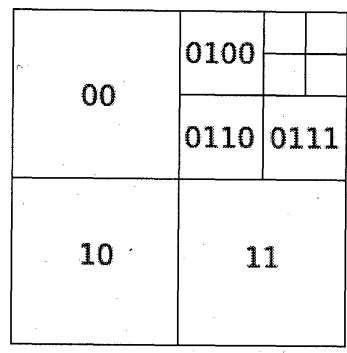

Fig. 2 Quad-tree mapping.

IP addresses. It means that a layer one grid stands for the 0.0.0.0/0 $0^{\dagger}$ network zone and that each of its quadrilaterals is associated with all the hosts whose IP addresses share the same first byte. From this state, a quadrilateral can be selected and then, a layer two grid replaces the previous layer one grid. For instance, if the selected quadrilateral corresponds to the first byte 192, the displayed layer two grid stands for the 192.0.0.0/8 network zone. Each quadrilateral of that new grid is associated with all the hosts whose IP address starts with the byte 192 and whose second byte is the value mapped on the quadrilateral. In the same way, a quadrilateral of that layer two grid can be selected, for instance 168 , and in this case, the layer three grid standing for the network zone 192.168.0.0/16 is displayed. Finally, a third byte can be selected and at this point, each quadrilateral of the resulting layer four grid stand for a unique host. To represent the single hosts (layer five), we use $256 \times 256$ grids whose quadrilaterals stand for TCP/UDP ports.

To map the IP addresses bytes on the first four layers grids, we chose to use a quad-tree scheme (Fig. 2) as it permits to gather hosts in subnets on the grids and thus to respect the network topology. Again, we wanted the representation of the network space to be as close as possible to the concepts used by system administrators. To map a byte, we consider its binary representation and decompose it into successive groups of two bits. We then cut the grid halfway along the rows and repeat the process along the columns to obtain four equal parts. The first group of two bits permits to select one of these parts. We then reiterate the cutting process on that selected part and use the second group of two bits to select one of the four resulting sub-parts. By reiterating four times this algorithm, we can map a byte to a precise quadrilateral of a $16 \times 16$ grid.

For the layer five grids, a mapping based on a quad-tree scheme does not introduce any interesting clustering of the ports. So we chose to use a different mapping based on a simple coordinate system. One axis (columns) corresponds to the port number modulo 256, the other axis (rows) corresponds to the port number divided by 256 .

We successively developed several visualizations for network traffic monitoring based on that interactive network space representation.

\section{2D Visualization}

\subsection{Description}

The visualization we initially imagined directly uses the previously introduced interactive grids to display network data. Depending on the layer it is set to, a grid stands for either a network zone or a single host. So, to display the network traffic exchanged between two network zones (or hosts), we chose to use groups of two grids. Within each group, one grid is considered as destination of the traffic (referred to as destination grid from now on) and the other as source of the traffic (referred to as source grid from now on). A grid can belong to several groups, for instance, it can act as a source grid for several destination grids.

To represent network traffic, we chose to draw lines for each packet captured. If a packet belongs to the traffic exchanged between the two network zones (or hosts) represented by a group of grids, a line is drawn between the corresponding quadrilaterals of the source and destination grids. A color code is used to indicate the protocol of the packet if it can be detected.

To represent network activity, we chose to use the color of the quadrilaterals of the grids. If a quadrilateral corresponds to a host, it is colored according to the number of packets received (for the destination grids) or sent (for the source grids) by this host. If it stands for a larger zone, it is colored according to the accumulated number of packets for the zone. And idem if the quadrilateral stands for a port. Regarding the color scale, we opted for the following rule: the higher the number of packets, the more vivid the color.

With this scheme, the user can clearly distinguish between incoming and outgoing network traffic on the visualization. It is also possible to monitor the internal traffic of a specific network zone by setting the two grids of a group to represent the same network zone.

\subsection{Evaluation and Limits}

We built a prototype based on the proposed 2D visualization and tested it displaying selected traffic from our corporate network (Fig. 3). 'For the prototype, we chose a configuration based on four grids grouped two by two. As the main priority of a system administrator is to watch his own network, we decided that two grids would represent the monitored network (referred to as static grids from now on). The other two grids would interactively be set by the user of the system (referred to as interactive grids from now on). We chose to position the grids side by side and to laid them out on the display as follows: the source static grid on the upper-left corner, the destination static grid on the lower-left corner, the source interactive grid on the lower-right corner and the destination interactive grid on the upper-right corner.

${ }^{\dagger}$ The A.B.C.D/n notation is a way to specify network zones, the first $n$ bits of the address identify the zone and the following bits, the hosts of that zone. 


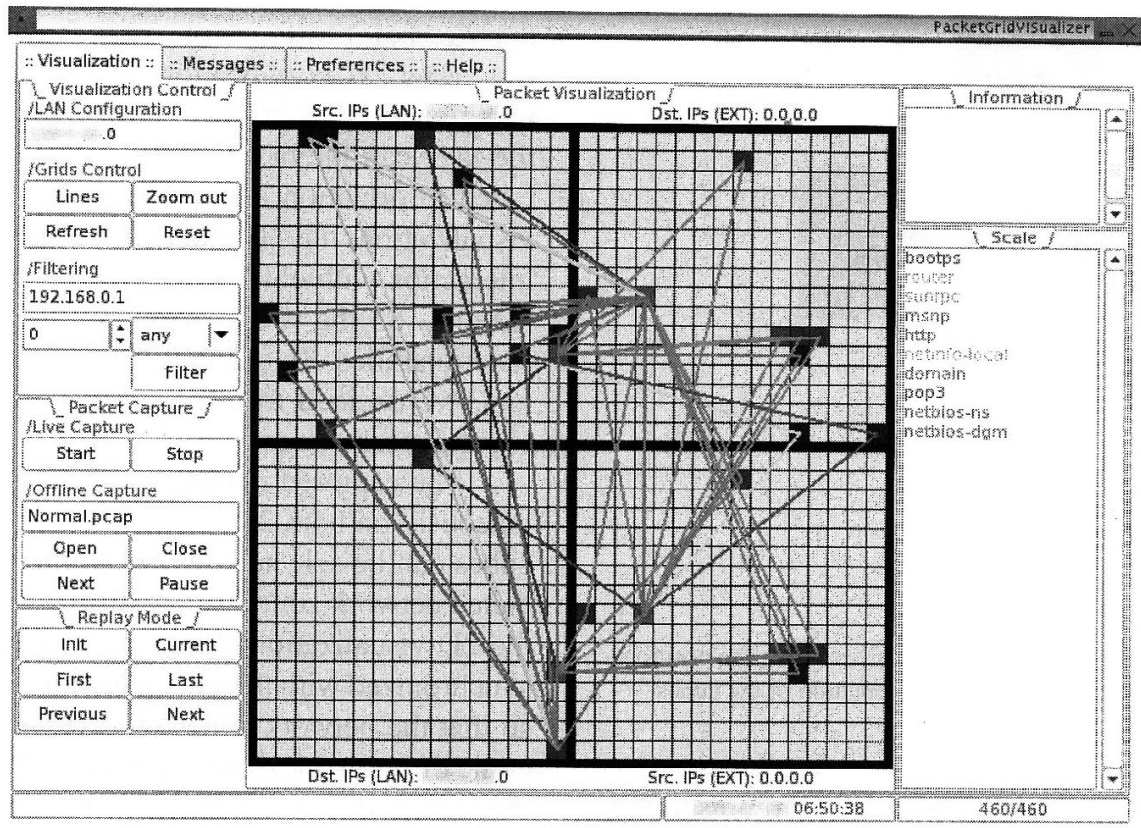

Fig. 3 Common traffic displayed on PGVis.

That structure enforces that lines are only drawn between adjacent grids, thus enhancing the clarity of the visualization.

Through that arrangement, the user is constantly provided with a view of the internal activity of the monitored network, as it is drawn between the two static grids (cf. our first requirement). He is also, at first, provided with a global view of the activity between the monitored network and the outside world (the interactive grids). In that initial state of the interactive grids (layer one), the data is quite aggregated as each quadrilateral stands for a large number of hosts. From that state, the user can modify the interactive grids to successively select more precise parts of the global network and, display the traffic between those zones and the network he is watching (cf. our second requirement). By selecting progressively smaller zones, the user can access more detailed information about the traffic as the quadrilaterals stand for less hosts. The user can then retrieve the exact location (IP address) of the communicating hosts and eventually analyze the distribution of the TCP/UDP ports they use to communicate (cf. our third requirement).

Using the prototype, we were able to identify more clearly the type of network traffic generated by each host of our corporate network. Visualization techniques made easier the overall comprehension of the network interactions in near real-time. We believe that this is the key to detect subtle or previously unknown attacks. Through the use of visualization, system administrators can learn how normal traffic looks like and so quickly detect deviations when they occur. For instance, they can spot patterns corresponding to potentially malicious activity such as multiple connections attempts to multiple local hosts from remote hosts (possible scanning activity), periodical bursts of activity from local hosts (possible botnet members), local hosts suddenly try- ing to connect to several other local hosts (possible worm propagation), connections from remote hosts to a local host on a port on which no service is supposed to be listening (possible compromised host), etc. Finally, it appeared to require less experience in network management and security to monitor the traffic with our prototype than with classical textual tools.

The results were encouraging, however, we noticed that the prototype had scalability and usability issues. The user was able to quickly distinguish global traffic patterns (through the implicit aggregation of the data provided by the network space representation) and then to analyze the associated traffic in depth by using the interactive hierarchical grid system to focus on different network zones. However that fine-grained analysis was quite time-consuming as the user could only visualize the network traffic exchanged between two network zones at a time (the local network zone and a selected external network zone). It was a substantial limitation as a system administrator usually needs to analyze the traffic involving a lot of different network zones.

To solve those issues, we tried to extend our visualization design and find a way to allow the display of the interactions between several network zones simultaneously. The simplest way was to increase the number of interactive grids, still, by using only 2D it was difficult to organize all the grids on the display. So we opted for the application of $3 \mathrm{D}$ visualization techniques.

\section{3D Visualization}

The $3 \mathrm{D}$ visualization was designed to be coherent with the $2 \mathrm{D}$ visualization. It is based on textured cubes laid out in a 3D space. Each cube stands for a network zone (that can be either $/ 0, / 8, / 16$ or $/ 24$ ) or a single host. Faces of a cube are 


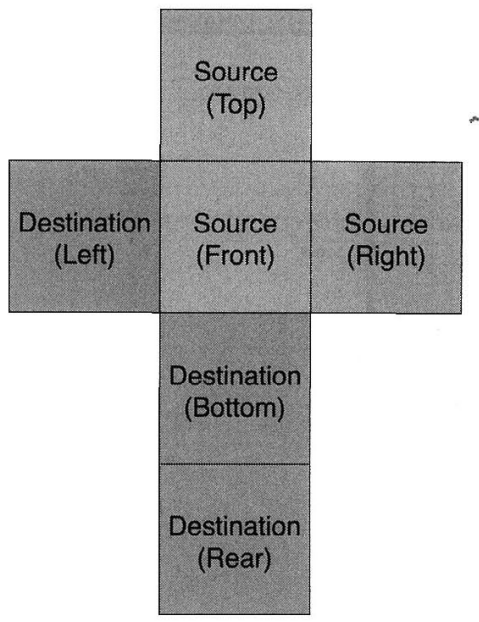

Fig. 4 Grid mapping on the cubes of the 3D visualization, the positions (between parenthesis) arc relative to the initial orientation of the cubes.

textured with grids (as described in Sect. 3), all representing the network zone or the host the cube is standing for. Three faces of each cube are mapped with the associated source grid (referred to as source faces from now on) and the other three faces are mapped with the destination grid (referred to as destination faces from now on). The arrangement of the grids is fixed for all cubes so that two opposite faces are always a source face and a destination face (Fig. 4).

The activity of the network zone or host associated with a cube is represented the same way as for the grids of the $2 \mathrm{D}$ visualization, encoded in the color of the quadrilaterals of the grids mapped on the faces. Two different color scales are used in order to differentiate the source and destination faces (red gradient for source faces and blue gradient for destination faces on the prototype).

A source face of a cube can be connected to a destination face of another cube. If so, the traffic from the network zone represented by the first cube to the one represented by the second cube is displayed between the two cubes. For each packet captured, a cone is drawn between the corresponding quadrilaterals on the connected source face and on the connected destination face. Using cones permits to display the direction of propagation of the packets. The time of capture of the packets is shown using a transparency effect. The older the packet, the more transparent the cone. In addition to that, the cones are colored using the same color scale as the one used for the lines of the 2D visualization.

\section{Linking the 2D and 3D Visualizations}

By using both visualizations separately we observed that they had distinct advantages. The $3 \mathrm{D}$ visualization provides an overview of the traffic between several selected network zones and hosts. The use of 3D brings flexibility compared to the $2 \mathrm{D}$ visualization. For instance, if the user wants to monitor an additional network zone, he only has to add the corresponding cubes to the visualization. Also, navigation in a $3 \mathrm{D}$ space is rather intuitive and users can easily modify the viewpoint to focus on parts showing suspicious behaviors. The $2 \mathrm{D}$ visualization provides a view of the packets being exchanged between two selected network zones and the user can interact with the grid system to get more detailed information about that traffic. Compared to the 3D visualization, the $2 \mathrm{D}$ one is more straightforward. Besides, interaction with objects is easicr in the $2 \mathrm{D}$ space than in the $3 \mathrm{D}$ one. So the two visualizations provide complementary functionalities. To enhance the overall efficiency of our system, we decided to use them both and link them through several mechanisms.

The captured network traffic is to be displayed simultaneously on both $2 \mathrm{D}$ and $3 \mathrm{D}$ visualizations. A pair of two cubes can be selected and the traffic between the two associated network zones is then displayed on the grid system of the 2D visualization. Similarly, a pair of two cubes can be synchronized with the grids positions of the $2 \mathrm{D}$ visualization so that the network zones represented by the grids also become the network zones represented by the cubes. It enables the user of the visualization system to analyze finely (using the $2 \mathrm{D}$ visualization) suspicious network traffic that he detects on the overview (3D visualization). He can use the grid system to "7oom" and select the involved network zones and hosts. If the suspicious network traffic happens to be malicious, the user can modify the settings of the overview to, for instance, add new cubes and synchronize them with the grid system. Doing so, he can kcep an cyc on that traffic and finely monitor it from the 3D visualization. Via this process, the user interactively creates a customized view according to his needs. He chooses the network zones that he wants to monitor, he is active. As for the 2D visualization, we developed a prototype, PGVis3D, based on that extended design (Fig. 5) and tested it on our corporate network.

\section{Prototypes}

\subsection{Implementation}

Regarding the implementation of the prototypes, we wanted our programs to run on a large number of operating systems and to use free open source libraries, especially for the visualization part, so that we could share our results more easily with the community.

We chose to write the prototypes in $\mathrm{C}$ language. We used the GTK +-2 .X toolkit [8] for the Graphic User Interface (GUI) and for the 2D visualization. As for the 3D visualization, we used GtkGLExt (OpenGL cxtension for GTK+-2.X) [9]. The network traffic capture and log processing are performed using the Libpcap library [10]. Both prototypes are known to compile and run on FreeBSD, GNU/Linux and Mac OS X.

\subsection{Discussion}

Throughout the conception of our prototypes, we tried to stick to the following principle: leave the interpretation of the data to the user and minimize the bias introduced by the 


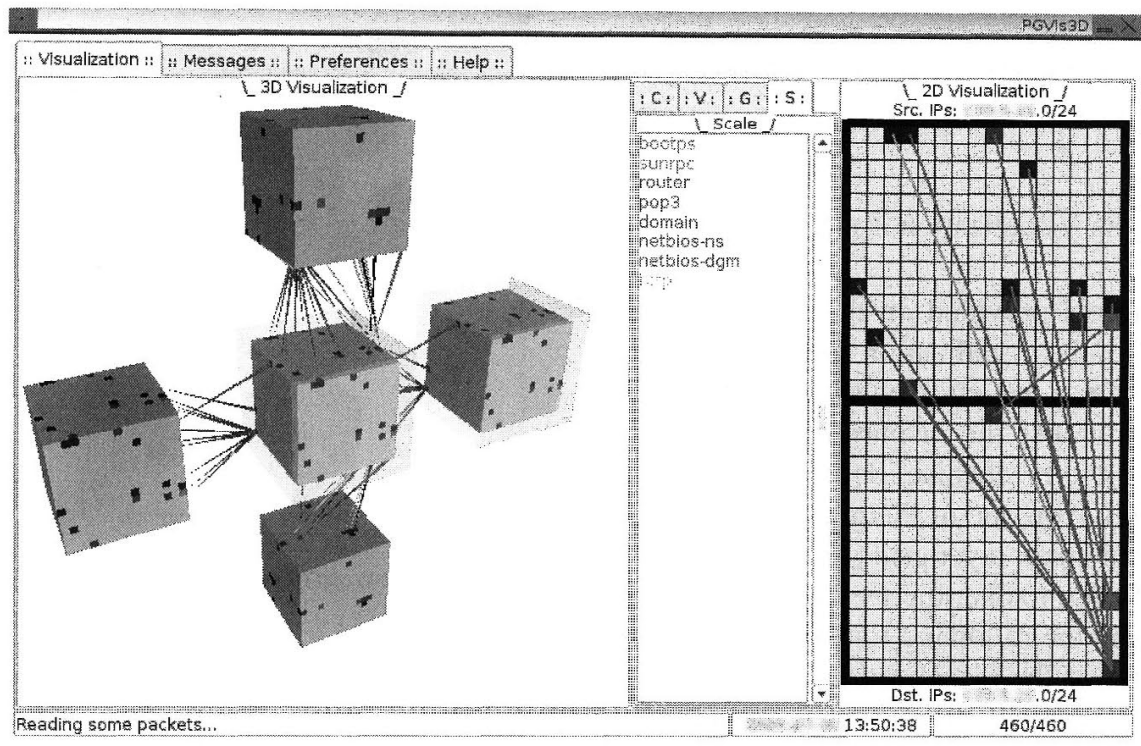

Fig. 5 Common traffic displayed on PGVis3D.

system relatively to that task. Some tools such as NIDSs heavily rely on automated algorithms to analyze network data, providing the user with their deductions. The main drawback of that approach is that those algorithms are still far from being perfect and sometimes guess wrong (e.g. false positives of NIDSs). The user is then required to go back to the original data and analyze it by hand to verify the deductions. We wanted to prevent such phenomenon from happening when using our tools.

To achieve that objective, we decided to keep the representation of the network data as straightforward as possible, trying not to distract the user with skewed information. We also focused on interactivity, providing the user with a panel of techniques for data exploration, in order to let him judge by himself. We do not advocate that system administrators should drop tools based on automated analysis as they have some strong points, but we believe that our tools can deliver some original insight into the data and may also be used to corroborate previous findings.

\subsection{Interface}

The interfaces of the two successive prototypes are quite similar as we reused large parts of the first prototype that we extended for the second one. We only had to develop the $3 \mathrm{D}$ visualization and integrate it to the interface, adding the necessary controls. The implementation of the core functionalities (network traffic capture, data storage and access, etc.) was kept almost identical. As we separated the data processing from the interface, the addition of another visualization would also be quite easy.

We chose to divide the interface of the prototypes gathering similar functionalities and we opted for a organization in four tabs:

- The "Visualization" tab: This is the main screen
(Figs. 3 and 5). From this part, the user can access the visualization zones and some related controls. For instance on PGVis3D, the 2D visualization zone is located on the right side of the screen, the $3 \mathrm{D}$ visualization zone on the left side and the controls on the center. These controls are also organized in tabs because of the space constraint:

- The "Capture Controls" tab ("C"), cf. the left panel on PGVis: It provides the controls to start a live packet capture, to open a previously saved network traffic dump file and to navigate in loaded data (replay mode).

- The "Visualization Controls" tab ("V"), cf. the left panel on PGVis: It provides controls to tweak the behavior of the visualization zones and to apply basic filters to the data.

- The "Grid \& Cube Controls" tab ("G", Fig. 7): It provides controls to select cubes and pairs of connected cubes. The selected cubes are highlighted on the display. The user can also synchronize the $2 \mathrm{D}$ and the $3 \mathrm{D}$ visualization zones.

- The "Scale" tab ("S", Fig. 5), cf. the right panel on PGVis: It provides the color scale (association between colors and protocols) used for the cones and the lines representing the packets.

- The "Messages" tab: Some status messages are printed under this tab. Headers of the displayed packets are also logged there so that the user can access detailed information if he wants to.

- The "Preferences" tab: The user can set some options and modify the behavior of the program. For instance, he can modify the color scale, set a Berkeley Packet Filter (BPF) type filter to display only matching packets, adjust the length of time that packets are displayed on the visualization zones, set the number of packets to 
store in memory, ...

- The "Help" tab: To display some basic information about the prototype.

\subsection{Interaction with the Visualization Zones}

In addition to the controls provided on the GUI, the user can interact directly with the visualization zones. Regarding the $2 \mathrm{D}$ visualization zone, a left-click on one of the quadrilaterals of the grids provokes the display of a new lower layer grid according to the mechanism described in Sect. 3 ("zoom in"). A right-click on one of the grids provokes the display of the associated upper layer grid ("zoom out"). Regarding the $3 \mathrm{D}$ visualization zone, the user can modify the viewpoint by performing the following transformations: rotating around the center of the visualization zone by translating the mouse while pushing its left button, zooming by translating the mouse while pushing its right button, panning by translating the mouse while pushing its middle button. It enables him to focus on desired information.

\subsection{Examples}

To evaluate the successive versions of our tool, we chose to visualize samples of the traffic generated by our corporate network $(100 \mathrm{Mb} / \mathrm{s}$ links). As we already had some knowledge about the expected network traffic characteristics, the process of determining how normal traffic looked like was simpler, and so did the detection of anomalies.

To conduct our tests with PGVis3D, we chose to use layouts of five or seven cubes for the $3 \mathrm{D}$ visualization zone. However, it can be modified and adapted to the user's needs. In the initial state, the cubes on the left, middle and right of the screen all stand for the local network (a / 24 network zone). The cubes on the top and bottom both stand for the global network (the $/ 0$ network zone). For the seven cubes layout, the additional cubes on the top-right and bottom-left both stand for a single host of the monitored network. Regarding the $2 \mathrm{D}$ visualization, the source grid is on the top and the destination grid on the bottom. We kept that configuration fixed for Figs. 5, 6 and 7. So the internal traffic of the local network is displayed between the left and middle cubes and between the middle and right cubes. The network traffic displayed between the bottom and middle cubes is the incoming traffic from the global network to the local network. The network traffic displayed between the middle and top cubes is the outgoing traffic from the local network to the global network. With the two additional cubes standing for the single host, the user can observe the traffic in both directions between this host and the local network, and between this host and the global network. Regarding Fig. 3 (PGVis), the interactive grids (on the right side) are set to the layer one of the hierarchy.

For Figs. 3 and 5, we used a small capture done on our local network from a connected host. On Fig. 5, the 2D visualization zone is set to display the internal traffic of the

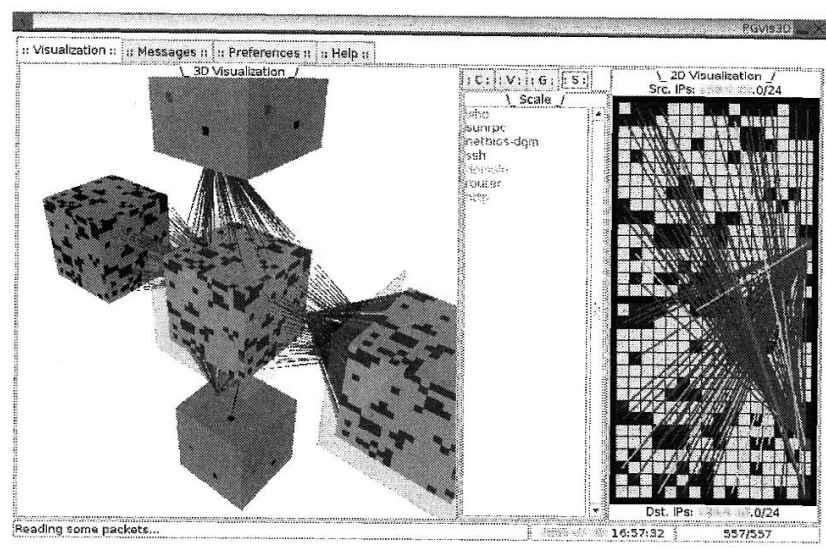

Fig. 6 Portsweep of our corporate network by an internal host, displayed on PGVis3D.

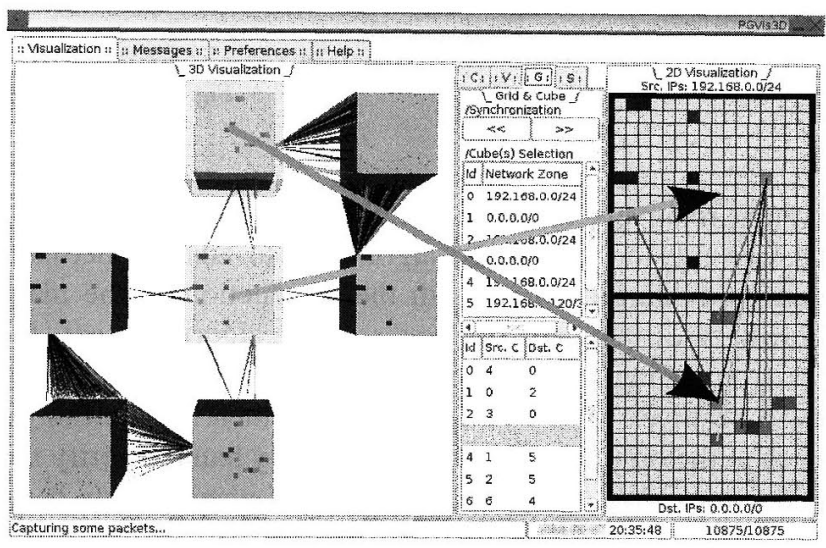

Fig. 7 Portscan of a local host, displayed on PGVis3D. Arrows were added to indicate the association between cubes and grids.

local network. The observed traffic is quite common, the local traffic is mainly constituted of broadcast activity. Regarding Fig. 6, we simulated a portsweep by probing all the local network on a specific port from a local host. The resulting traffic is highly noticeable on the $3 \mathrm{D}$ visualization zone, with cones drawn between one quadrilateral of a face and multiple quadrilaterals of the opposite face. This pattern can be seen between all the connected faces as, in the chosen layout, the local network (left, middle and right cubes) is also part of the global network (top and bottom cubes). The 2D visualization zone enables the user to get the exact source of that attack and the color scale provides information about the target (port associated with SSH service). For Fig. 7, we used the seven cubes layout. The traffic displayed contains a portscan of the host mapped on the additional cubes. That portscan is also highly noticeable with cones drawn toward a lot of quadrilaterals of these two cubes. A remote host is trying to connect successively to several ports of the monitored host. On that figure, the transparency effect is activated and we can see the cones progressively vanishing on the $3 \mathrm{D}$ visualization zone. The advantage is that the user can focus on recent events and watch them nearly as they occur. For instance, while monitoring a host of our cor- 
porate network with PGVis3D we suddenly noticed a cone standing for a SSH connection attempt toward it. This event could have been insignificant but to our knowledge no one was supposed to connect to that host during that time frame, so it became highly suspicious. An inspection of the system logs of the affected host later confirmed that it was under SSH brute force attack.

\subsection{Performances}

The main bottleneck in regard to the performances of PGVis $3 \mathrm{D}$ is the drawing of the $3 \mathrm{D}$ visualization zone which is quite resource consuming. This is especially true when using the prototype in live packet capture mode as it is hard to control the number of objects constituting the 3D scene. To each additional packet captured corresponds a new object to display, illuminate and animate. The duration packets are to be displayed after capture can be adjusted to reduce the number of simultaneously visible objects, thus enabling the real-time processing of the traffic exchanged by several hosts of a standard network. Performances get better when using 3D hardware acceleration.

However, even with PGVis, dealing with high speed networks in live packet capture mode is still an issue as both system and users can become overloaded, unable to keep up with the incoming flow of information. Such traffic should be handled offline, by making use of the replay functionality of the prototypes. Both prototypes can read network traffic dump files (Pcap format [10]). These files can be loaded entirely into memory or browsed sequentially, allowing the processing of very large dumps without risking memory exhaustion. By controlling the replay rate, the user can then adjust the amount of traffic to simultaneously display, matching his processing capabilities. Our tests indicate that the prototypes can generally handle the replay rates requested by users.

\subsection{Envisioning}

One of the main strengths of our design is its modularity, it can be adapted to a wide range of network topologies. For instance, Figs. 8 and 9 describe some basic ways of using PGVis3D to monitor simultaneously several network zones along with the traffic they exchange. Such layouts could easily be extended to handle more network zones, a limitation being the display size allocated to the tool. Another point to consider, which is still an open research issue, is the efficient aggregation of network traffic captured at different locations for centralized processing.

\section{Related Work}

Our research is based on the application of techniques from the field of information visualization to the network traffic monitoring task. Several surveys exist regarding the characteristics of available basic visualization techniques.

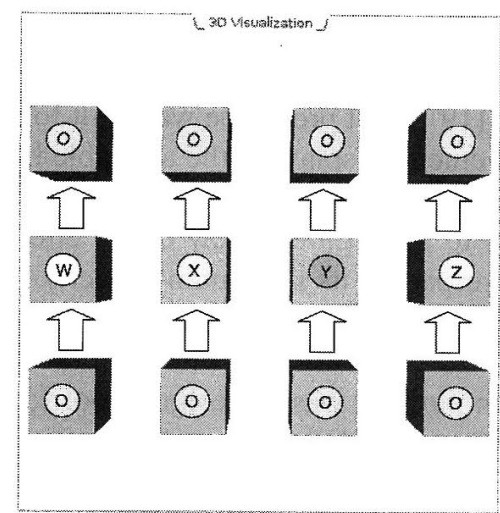

Fig. 8 PGVis3D layout to monitor four local network zones (W, X, Y and $Z$ ) and the traffic they exchange with the outside world $(\mathrm{O}, / 0$ network zone). Arrows indicate how cubes are connected, and thus what traffic is displayed.

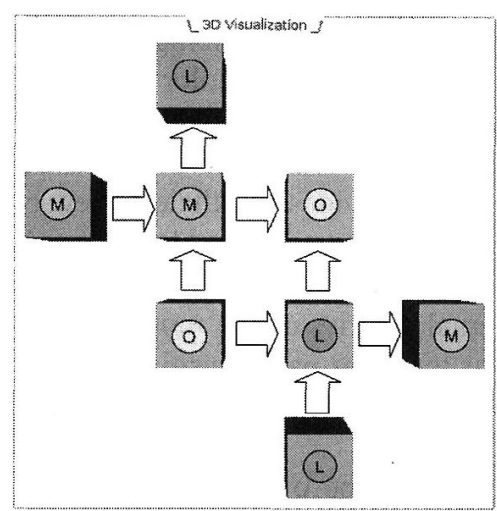

Fig.9 PGVis3D layout to monitor all traffic exchanged by two local network zones ( $\mathrm{L}$ and $\mathrm{M}, \mathrm{O}$ being the $/ 0$ network zone).

Shneiderman proposed such a taxonomy sorting visualization techniques not only according to the type of data to visualize but also according to the task to be accomplished [7]. It provides a large panel of available techniques that can be combined to make complex visualization systems. Chi proposed a taxonomy based on a different approach, he classified the visualization techniques not only according to the type of the data to visualize but also according to the processing steps to achieve the visualization of that data [11]. Toolkits were developed to simplify the use of these classical visualization techniques like Prefuse by Heer et al. [12] and The InfoVis Toolkit (IVTK) by Fekete [13]. For global surveys of the field of information visualization, the reader can refer to the works of Spence [14] and Tufte [15]-[17].

Several tools have been proposed to monitor networks graphically. Most of them are based on $2 \mathrm{D}$ visualization zones. EtherApe [18] is a free open source tool built to display network activity between hosts. It displays hosts as nodes laid out in a circle. Network activity is symbolized by the thickness of the links between the nodes and colors are used to represent the recognized protocols. This tool is wellsuited to monitor a limited number of hosts. However, as the number of hosts increases, the visualization zone becomes 
difficult to interpret. Erbacher also proposed a visualization system focusing on the activity of the hosts of a limited network zone [19]. That prototype was built for network resource management, displaying the available bandwidth on each network link through the use of glyphs. That tool enables the user to get a global view of the effective load of a network in respect to its maximum capacity. However, as with EtherApe the number of hosts that can be monitored is limited.

Our main purpose was to design a tool scaled for system administrators so that they could monitor the activity of their networks. VISUAL [20] from Ball et al. is such a tool. It focuses on the display of the communications between the local network that the system administrator wants to monitor and the outside world. The monitored network is represented by a grid whose quadrilaterals each stand for a host. External hosts are represented as markers laid around the grid. Connections between internal and external hosts are symbolized by segments linking the markers and the associated quadrilaterals. The color of the segments indicates if the communication is bidirectional or unidirectional. The communication bandwidth is encoded in the size of the markers. VISUAL provides an efficient view of the interactions between the monitored network and the outside but the number of external hosts that can be represented is limited. Also, the internal activity of the monitored network is not displayed. Our tools are partly inspired from the design of VISUAL, we tried to extend it to handle the monitoring of the local network.

The Spinning Cube of Potential Doom [21] is a tool using $3 \mathrm{D}$ visualization techniques to display data regarding established TCP connections and connection attempts. That data is mapped in a cube, the $\mathrm{x}$-axis of the cube stands for the local network's IP address space, the $\mathrm{z}$-axis for the global IP address space and the y-axis for the TCP ports. Each TCP connection is displayed by a dot. This dot is white for an established connection and colored according to the port number for a connection attempt. In this way, connection attempts are emphasized and portscans are easy to detect as they appear as large colored zones on the display. However, after the detection, the exact origins of those attacks are difficult to retrieve using this tool.

Regarding the use of 3D visualization techniques, Oline and Reiners [22] proposed several designs for network security using either raw network traffic or NIDSs output. They were able to detect several anomalies with each visualization tools that they implemented according to their designs. However, with some of their tools, it is difficult to get detailed information about the traffic displayed such as for instance the addresses of the hosts involved. Also, the interaction with the visualization zones of each tool is limited to modifications of the user's viewpoint using camera transformations.

We chose to design our tools so that network topology was apparent on the visualization zones. Some other tools adopted a different approach. PortVis [23] from McPherson et al. is such a tool and has intentionally been designed to provide as little information as possible about the structure and topology of the networks to analyze. Indeed, these details can be quite sensitive. To cope with that issue, PortVis uses a group of network traffic attributes really limited and aggregated hour per hour for each TCP/UDP port. The main screen of that tool is composed of a $256 \times 256$ grid whose dots each represent a port. The y-axis corresponds to the port number modulo 256 and the $\mathrm{x}$-axis corresponds to the port number divided by 256 , a mapping we also used for the layer five grid of our tool. A color scale is used for the dots to display information about the selected network traffic attribute. Among the tools not built on a metaphor of the network topology, several use the classical parallel coordinates visualization technique to display network traffic data [24], [25].

Oberheide et al. [26] recently proposed a tool for network traffic monitoring which shares some basic principles with our tools (use of a quad-tree scheme and combination of $2 \mathrm{D}$ and $3 \mathrm{D}$ visualization techniques). However, in their tool, interactivity with the visualization zones is limited to modifications of the user's viewpoint, they mainly focused on the presentation of the data. Whereas in our tools, we provide a way to visually control the $3 \mathrm{D}$ visualization zone through actions on the interactive $2 \mathrm{D}$ visualization zone and so, enable the user to perform fine-grained analysis of the data. Also, their 3D representations of the data do not allow the user to focus on the activity and traffic generated by specific network zones.

Finally, Conti et al. [27] provide a survey of possible attacks against visualization systems. We tried to take into account their advises while developing our tools to make them less vulnerable to those attacks.

\section{Conclusion}

In this paper, we presented some original visualization techniques that we successively developed to simplify the network traffic monitoring task and the tools we built using these techniques. We started by analyzing the needs of the system administrators managing our corporate network and also previously proposed tools using visualization techniques for network security. From that analysis, we designed a hierarchical representation of the network space based on interactive grids. The initial prototype that we developed around that representation proved to be useful for fine-grained analysis of the network traffic and for anomaly detection. However, after a period of testing we noticed that it had several limitations. In particular, it was only possible to monitor the traffic between two network zones at a time and monitoring complex traffic was difficult to handle as it required an intensive use of the interactive grid system. As a consequence, we developed a second visualization design trying to combine 2D and 3D visualizations of the network traffic in order to solve those issues. Through the tests conducted with the associated prototype, the proposed updated visualization design seemed to be more scalable than the previous one. Also, combining both $2 \mathrm{D}$ and $3 \mathrm{D}$ visual- 
izations increased the overall usability of the prototype. A lesson we learned from that research, is of the importance of providing several linked view of the data.

The current prototypes are usable and include all the basic functionalities corresponding to the proposed visualization designs. The first prototype is quite complete, so we will focus our work on the second one to extend its functionalities and increase its usability. We intend to conduct an user evaluation of our tools once they are finalized. We also plan to release them to the research community so that more system administrators could try our tools. Finally, in relation with this research we are interested in the adaptation of our visualization designs to the IPv6 address space as it is to replace the IPv4 system. We are also considering the adaptation of our prototypes to handle network flow data (e.g. NetFlow [28]) in addition to raw packet data. The use of such aggregated format could make even easier the processing of huge amounts of traffic.

\section{Acknowledgments}

The authors would like to thank Yoshiaki Kasahara for his valuable comments. Support for this work is partially provided by National Institute of Information Communications and Technology (NICT), Japan, as a visualization technology research for the nicter Incident Analysis Center project.

\section{References}

[1] E. Le Malécot, M. Kohara, Y. Hori, and K. Sakurai, "Grid based network address space browsing for network traffic visualization," Proc. 7th IEEE Information Assurance Workshop (IAW'06), pp.261-267, IEEE Computer Society, Washington, DC, USA, 2006.

[2] E. Le Malécot, M. Kohara, Y. Hori, and K. Sakurai, "Interactively combining 2D and 3D visualization for network traffic monitoring," Proc. 3rd International Workshop on Visualization for Computer Security (VizSEC'06), pp.123-127, ACM Press, New York, NY, USA, 2006.

[3] R. Pang, V. Yegneswaran, P. Barford, V. Paxson, and L. Peterson, "Characteristics of Internet background radiation," Proc. 4th ACM SIGCOMM Conference on Internet Measurement (IMC'04), pp.2740, ACM Press, New York, NY, USA, 2004.

[4] "Tcpdump." http://www.tcpdump.org

[5] "Wireshark." http://www.wireshark.org

[6] D. Schweitzer and L. Baird, "Discovering an RC4 anomaly through visualization," Proc. 3rd International Workshop on Visualization for Computer Security (VizSEC'06), pp.91-94, ACM Press, New York, NY, USA, 2006.

[7] B. Shneiderman, "The eyes have it: A task by data type taxonomy for information visualizations," VL, pp.336-343, 1996.

[8] "GTK+." http://www.gtk.org

[9] "GtkGLExl." http://gtkglext.sourceforge.net

[10] "Libpcap." http://www.tcpdump.org

[11] E.H. Chi, "A taxonomy of visualization techniques using the data state reference model," Proc. IEEE Symposium on Information Vizualization 2000 (INFOVIS'00), p.69, IEEE Computer Society, Washington, DC, USA, 2000.

[12] J. Heer, S.K. Card, and J.A. Landay, "Prefuse: A toolkit for interactive information visualization," Proc. SIGCHI Conference on Human Factors in Computing Systems (CHI’05), pp.421-430, ACM Press, New York, NY, USA, 2005.

[13] J.D. Fekete, "The InfoVis toolkit," Proc. IEEE Symposium on Infor- mation Visualization (INFOVIS'04), pp.167-174, IEEE Computer Society, Washington, DC, USA, 2004.

[14] R. Spence, Information Visualization, Pearson Addison Wesley, 2000.

[15] E.R. Tufte, The Visual Display of Quantitative Information, Second Edition, Graphics Press, 2001.

[16] E.R. Tufte, Visual Explanations: Images and Quantities, Evidence and Narrative, Graphics Press, 1997.

[17] E.R. Tufte, Envisioning Information, Graphics Press, 1990.

[18] "EtherApe." http://etherape.sourceforge.net

[19] R. Erbacher, "Visual traffic monitoring and evaluation," Proc. Conference on Internet Performance and Control of Network System II, pp.153-160, 2001.

[20] R. Ball, G.A. Fink, and C. North, "Home-centric visualization of network traffic for security administration," Proc. 2004 ACM Workshop on Visualization and Data Mining for Computer Security (VizSEC/DMSEC'04), pp.55-64, ACM Press, New York, NY, USA, 2004.

[21] S. Lau, "The spinning cube of potential doom," Commun. ACM, vol.47, no.6, pp.25-26, 2004.

[22] A. Oline and D. Reiners, "Exploring three-dimensional visualization for intrusion detection," Proc. IEEE Workshops on Visualization for Computer Security (VizSEC'05), p.14, IEEE Computer Society, Washington, DC, USA, 2005.

[23] J. McPherson, K.L. Ma, P. Krystosk, T. Bartoletti, and M. Christensen, "PortVis: A tool for port-based detection of security events," Proc. 2004 ACM Workshop on Visualization and Data Mining for Computer Security (VizSEC/DMSEC'04), pp.73-81, ACM Press, New York, NY, USA, 2004.

[24] P. Hertzog, "Visualizations to improve reactivity towards security incidents inside corporate networks," Proc. 3rd International Workshop on Visualization for Computer Security (VizSEC'06), pp.95102, ACM Press, New York, NY, USA, 2006.

[25] C.P. Lee and J.A. Copeland, "Flowtag: A collaborative attackanalysis, reporting, and sharing tool for security researchers," Proc. 3rd International Workshop on Visualization for Computer Security (VizSEC'06), pp.103-108, ACM Press, New York, NY, USA, 2006.

[26] J. Oberheide, M. Goff, and M. Karir, "Flamingo: Visualizing Internet traffic," Proc. 10th IEEE/IFIP Network Operations \& Management Symposium (NOMS'06), pp.150-161, IEEE Computer Society, Washington, DC, USA, 2006.

[27] G. Conti, M. Ahamad, and J. Stasko, "Attacking information visualization system usability overloading and deceiving the human," Proc. 2005 Symposium on Usable Privacy and Security (SOUPS'05), pp.89-100, ACM Press, New York, NY, USA, 2005:

[28] B. Claise, "RFC 3954: Cisco systems NetFlow services export version 9," 2004.

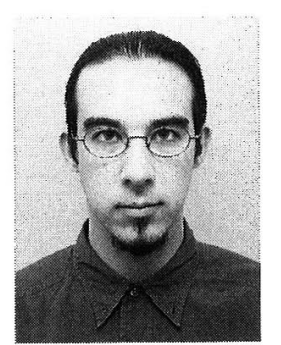

Erwan Le Malécot received both Engineering degree and research Master's degree in computer science from Supélec (one of the French "Grandes Écoles", specialized in Electrical Engineering and Computer Science) in 2006. He then entered the Graduate School of Information Science and Electrical Engineering, Kyushu University in 2007 and is currently studying toward D.E. degree. His research interests includc network security and information visualization. 


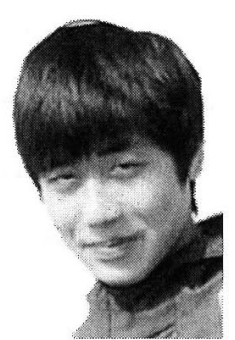

Masayoshi Kohara received B.E. degree from the Faculty of Engineering, Kyushu University and M.E. degree from the Graduate School of Information Science and Electrical Engineering, Kyushu University in 2005 and 2007 respectively. His research interests include network traffic analysis and network management. He is now employed by NTT DATA Corporation since 2007.

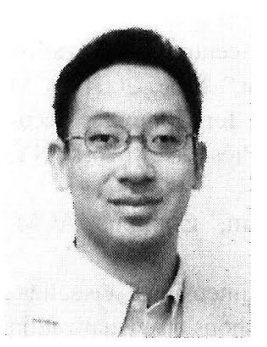

Yoshiaki Hori received B.E., M.E., and D.E. degrees on Computer Engineering from the Kyushu Institute of Technology, Iizuka, Japan in 1992, 1994, and 2002, respectively. From 1994 to 2003, he was Research Associate at Common Technical Courses, Kyushu Institute of Design, Fukuoka. From 2003 to 2004, he was Research Associate at the Department of Art and Information Design, Kyushu University, Fukuoka. Since March 2004, he has been Associate Professor at the Department of Computer Science and Communication Engineering, Kyushu University. His research interests include network security, network architecture, and performance evaluation of network protocols on various networks. He is a member of IEEE, ACM, and IPSJ.

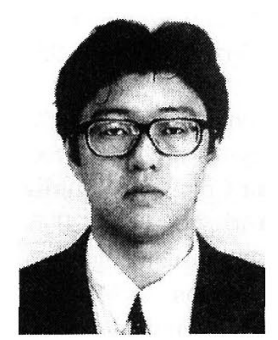

Kouichi Sakurai received the B.S. degree in mathematics from the Faculty of Science, Kyushu University and the M.S. degree in applied science from the Faculty of Engineering, Kyushu University in 1986 and 1988 respectively. He had been engaged in the research and development on cryptography and information security at the Computer and Information Systems Laboratory at Mitsubishi Electric Corporation from 1988 to 1994 . He received D.E. degree from the Faculty of Engineering, Kyushu University in 1993. Since 1994 he has been working for the Department of Computer Science of Kyushu University as Associate Professor, and now he is Full Professor from 2002. His current research interests are in cryptography and information security. Dr. Sakurai is a member of the Information Processing Society of Japan, the Mathematical Society of Japan, ACM and the International Association for Cryptologic Research. 